\title{
Multiples Myelom - ASCO 2016 - Bestätigung der ASZT und neue Daten zur systemischen Therapie
}

\section{Stammzelltransplantation bleibt Standard in der Erstlinientherapie des multiplen Myeloms}

Die Hochdosistherapie mit autologer Stammzelltransplantation (ASZT) ist etablierter Standard in der Erstlinientherapie fitter Myelom-Patienten unter 65 Jahren. In Anbetracht der hohen Wirksamkeit der neuen Substanzen stellt sich nunmehr die Frage, ob eine ASZT bei jungen Patienten noch erforderlich ist. Die Phase-III-Studie EMN02/H095 MM [1] verglich daher die primäre ASZT mit einer Bortezomib-haltigen Chemotherapie bei insgesamt 1266 neu diagnostizierten Myelom-Patienten.

Alle Patienten erhielten zunächst eine Induktionstherapie mit VCD (Bortezomib, Cyclophosphamid, Dexamethason) und wurden dann randomisiert zu entweder 4 Zyklen VMP (Bortezomib, Melphalan, Prednison) oder ASZT mit hochdosiertem Melphalan. Danach folgte eine zweite Randomisierung zwischen Konsolidierung mit 2 Zyklen VRD (Bortezomib, Lenalidomid, Dexamethason) versus keine Konsolidierung. Alle Patienten erhielten eine Erhaltungstherapie mit Lenalidomid bis zur Progression.

Die geplante Zwischenanalyse zeigte für die ASZT ein signifikant besseres progressionsfreies Überleben (PFS; primärer Endpunkt) verglichen mit VMP (HR 0,73; $\mathrm{p}=0,003)$. So lag die progressionsfreie Rate nach 3 Jahren bei 55,2\% im Transplantationsarm gegenüber 43,2\% im VMP-Arm. Während das mediane PFS im Transplantationsarm noch nicht erreicht war, betrug es im VMP-Arm 44 Monate. Der Vorteil zeigte sich dabei in allen untersuchten Risikogruppen (stratifiziert nach ISS-Stadium und zytogenetischem Risikoprofil). Zudem war die Anzahl sehr guter Teilremissionen ('very good partial response', VGPR) im Transplantationsarm signifikant höher als im VMP-Arm (43,2\% vs. 30,4\%; $<$ <,001). Eine multivariate Analyse aller Patienten bestätigte die ASCT als unabhängigen Faktor für eine Verlängerung des PFS. Die Daten zum Gesamtüberleben sind noch nicht reif; ein Unterschied ist bisher nicht vorhanden [1].

\section{Zusammenfassender Kommentar}

Auch in der Ära der neuen Substanzen kann derzeit auf die Hochdosistherapie mit ASZT nicht verzichtet werden. Trotz bes- serem Ansprechen und signifikant längerem PFS konnte bisher aber noch kein Gesamtüberlebensvorteil für die ASZT gezeigt werden.

\section{Dreifachkombination mit Daratumumab beim rezidivierten/refraktären multiplen Myelom}

Daratumumab wurde kürzlich von der EMA als Monotherapie bei stark vorbehandelten Patienten mit rezidiviertem/refraktärem multiplem Myelom zugelassen. Aufgrund der relativ guten Verträglichkeit des CD38-Antikörpers bieten sich eine Reihe von Kombinationsmöglichkeiten an, die derzeit in mehreren Studien geprüft werden; so z.B. in der Phase-III-Studie CASTOR [2], die beim ASCO 2016 präsentiert wurde.

Die CASTOR-Studie untersuchte den Nutzen der Zugabe von Daratumumab zu einer in der Rezidivsituation etablierten Standardtherapie mit Bortezomib/Dexamethason bei 498 vorbehandelten Myelom-Patienten (median 2 Vortherapien, Range 1-10). Die Patienten erhielten in beiden Studienarmen jeweils 8 Zyklen, danach wurde im Daratumumab-Arm der CD38-Antikörper als Erhaltungstherapie weitergeführt.

Bereits nach einer kurzen Nachbeobachtungszeit von median 7,4 Monaten zeigte sich ein signifikanter Vorteil im PFS (primärer Endpunkt) zugunsten der Zugabe von Daratumumab (Median noch nicht erreicht vs. 7,2 Monate; HR 0,39; $\mathrm{p}<0,0001$ ). Der Unterschied beim 1-Jahres-PFS betrug $60,7 \%$ gegenüber $26,9 \%$ bei Bortezomib/Dexamethason alleine, was einer Verbesserung um $61 \%$ entspricht. Der Vorteil im PFS war in allen klinisch relevanten Subgruppen erkennbar, besonders aber bei Patienten mit ISSStadium I und bei Patienten mit nur einer Vortherapie. Daratumumab verbesserte zudem die Gesamtansprechrate signifikant ( $83 \%$ vs. 63\%; p < 0,0001) und verdoppelte die Rate kompletter Remissionen (19\% vs. 9\%; $\mathrm{p}=0,0012$ ) sowie die Rate an sehr guter partieller Remission (59\% vs. 29\%; p < 0,0001). Zu den klinisch relevanten Nebenwirkungen im Zusammenhang mit Daratumumab zählten Infusionsreaktionen, die vor allem bei der ersten Applikation auftraten und meist vom Grad 1 oder 2 waren. Andere unerwünschte Wirkungen traten in beiden Gruppen ähnlich häufig auf und entsprachen den bisherigen Erfahrungen mit Daratumumab [2]. 


\section{Zusammenfassender Kommentar}

Die Dreifachkombination mit Bortezomib/Dexamethason plus Daratumumab stellt einen potentiellen neuen Standard in der Rezidivtherapie des multiplen Myeloms dar, mit einer bisher unerreichten Reduktion des Progressionsrisikos um 61\% im Vergleich zu Bortezomib/Dexamethason. Offen ist der Effekt auf das Gesamtüberleben.

\section{Lenalidomid-Erhaltung verbessert Gesamtüberleben bei multiplem Myelom}

Eine Lenalidomid-Erhaltungstherapie nach ASZT verringert das Progressionsrisiko bei Patienten mit multiplem Myelom signifikant; die Daten zum Gesamtüberleben waren bisher nicht eindeutig. Mit der vorliegenden prospektiv geplanten Metaanalyse konnte nun auch ein längeres Gesamtüberleben durch die Lenalidomid-Erhaltungstherapie bestätigt werden [3].

Die Metaanalyse zum Gesamtüberleben beruht auf 3 großen Phase-III-Studien mit insgesamt 1209 Patienten, die eine Lenalidomid-Erhaltungstherapie versus Placebo oder keine Erhaltung im Anschluss an eine ASZT-Erstlinientherapie untersuchten: die US-amerikanische CALGB-Studie, die französische IFM 2005-02-Studie und die italienische GIMEMA-RVMM-PI-209Studie.
Bei median 80 Monaten Nachbeobachtungszeit wiesen die Patienten im Lenalidomid-Erhaltungstherapiearm ein signifikant besseres Gesamtüberleben auf als Patienten ohne Erhaltungstherapie (medianes Gesamtüberleben nicht erreicht vs. 86 Monate; $\mathrm{p}=0,001$ ). Das 7-Jahres-Gesamtüberleben betrug $62 \%$ versus $50 \%$. Damit verringerte die Lenalidomid-Erhaltung das Sterberisiko um 26\% (HR 0,74), was einer Verbesserung des mittleren Überlebens um geschätzt 2,5 Jahre entspricht. Im Lenalidomid-Erhaltungsarm wurde allerdings ein etwa um das 2-fach erhöhes Risiko für Zweitmalignome beobachtet, wobei die absolute Zahl der Fälle gering war und somit der Überlebensvorteil das Risiko überwiegt [3].

\section{Zusammenfassender Kommentar}

Eine Lenalidomid-Erhaltungstherapie kann aufgrund dieser Daten für die meisten Patienten als Standard nach Erstlinien-ASZT angesehen werden. Zu klären ist sicherlich noch die Frage nach der optimalen Dauer der Erhaltungstherapie.

Gerhard Emrich in Zusammenarbeit mit Prof. Hartmut Goldschmidt, Heidelberg.

\section{References}

1 Cavo $M$ et al.: Upfront autologous stem cell transplantation versus novel agent-based therapy for multiple myeloma: a randomized phase III study of the European Myeloma Network (EMN02/HO95 MM trial). ASCO 2016, abstr 8000
2 Palumbo A et al.: Phase III randomized controlled study of daratumumab, bortezomib, and dexamethasone (DVd) versus bortezomib and dexamethasone (Vd) in patients (pts) with relapsed or refractory multiple myeloma (RRMM): CASTOR study. ASCO 2016, LBA4.
3 Attal $\mathrm{M}$ et al.: Lenalidomide (LEN) maintenance (MNTC) after high-dose melphalan and autologous stem cell transplant (ASCT) in multiple myeloma (MM): a meta-analysis (MA) of overall survival (OS). ASCO 2016, abstr 8001 . 